\title{
Evaluation of the measurement uncertainty of the stiffness modulus: test case of indirect tensile on cylindrical specimens
}

\author{
Hicham Mezouara ${ }^{1, *}$, Latifa Dlimi ${ }^{1}$, Abdelouahhab Salih ${ }^{2}$, Mohamed Afechcar $^{3}$, and Houcine Zniker ${ }^{1}$ \\ ${ }^{1}$ Faculty of Science Department of Physics Ibn Tofail University Kenitra, Morocco \\ ${ }^{2}$ Higher Normal School of Technical Education, Department of Mechanical Engineering - E.N.S.E.T. B.P. 6207 Rabat, Morocco \\ ${ }^{3}$ National Center for Studies and Road Research CNER/DRCR BP 6226, Rabat-Instituts Rabat, Morocco
}

Received: 2 June 2018 / Accepted: 3 March 2021

\begin{abstract}
This study treats the measurement uncertainties that we can find in the stiffness modulus of the bituminous test. We present all the sensors installed on rigidity modulus measurement chains and also their uncertainty ranges. Several parameters influence the rigidity module's value, such as the parameters related to experimental conditions, and others are rather connected to the equipment's specification, which are the speed, the loading level, the temperature, the tested sample dimension, and the data acquisition, etc. All these factors have a great influence on the value of the modulus of rigidity. To qualify the uncertainty factors, we used two approaches: the first one is made by following the method described by the GUM (Guide to the expression of uncertainty in measurement), the second approach based on the numerical simulation of the Monte Carlo. The two results are then compared for an interval of confidence of $95 \%$. The paper also shows the employment of the basic methods of statistical analysis, such as the Comparing of two variances. Essential concepts in measurement uncertainty have been compiled and the determination of the stiffness module parameters are discussed. It has been demonstrated that the biggest source of error in the stiffness modulus measuring process is the repeatability has a contribution of around $45.23 \%$.
\end{abstract}

Keywords: Stiffness modulus / uncertainty / propagation of variances / GUM / Monte-Carlo

\section{Introduction}

Results should not be presented as a single number but similarly to a continuous random variable so as an interval named the confidence interval. Thus, with the specified probability that is assigned to this interval, it can be assumed that the resulting measurement value is contained within this interval, providing that the measurement and the accuracy analysis have been properly conducted. Calculating measurement uncertainty is related to such notions as tolerance and required measurability. Tolerance is a denominate number that represents the difference between the maximal and minimal values of a certain property. It determines the interval, in which the real values of the specified property for the individual exemplars of the produced items should be contained.

There are requirements for test laboratories to evaluate and report the uncertainty associated with their test results. Such requirements may be demanded by a customer who wishes to know the bounds within which

\footnotetext{
* Corresponding author: hicham_dess@yahoo.fr
}

the reported result may be reasonably assumed to lie, or the laboratory itself may wish to understand which aspects of the test procedure have the greatest effect on results so that this may be monitored more closely or improved.

Bituminous mixtures are highly heterogeneous material, which is one of the reasons for high measurement uncertainty when subjected to tests. The results of such tests are often unreliable, which may lead to making bad professional judgments. They can be avoided by carrying out reliable analyses of measurement uncertainty adequate for the research methods used and conducted before the actual research is done. This paper presents the calculation of measurement uncertainty using as an example the determination of the stiffness Modulus of the asphalt mixture, which, in turn, was accomplished using the indirect tension method.

In the test laboratories, the realization of the tests implies the use of the most effective means in the production and in the control.

However, knowledge of measurement uncertainties in control and metrology operations remains a major problem that is still poorly understood. 
The parameters that can modify the results of these rigidity modulus tests are too many to count such as the parameters related to the test conditions and others, rather, related to the specification of the equipment which is the speed and the level of loading, the temperature, the size of the sample tested and the data acquisition, etc. All these factors have a great influence on the value of the modulus of rigidity.

To qualify the uncertainty factors, we used two approaches: Approach 1 is made by following the approach described by the GUM (Guide to the expression of uncertainty in measurement), the approach 2 by the numerical simulation of the Monte Carlo. The two results are then compared for an interval of confidence of $95 \%$.

The indirect tensile test on cylindrical test specimens of the stiffness Modulus is based on standardization EN 12697-26 "Bituminous mixtures - Essay methods for hot hydrocarbon mixtures - Part 26: Rigidity modulus", appeared in 2004 [1-3]. Of the five annexes corresponding to as many test methods, we are interested here in Appendix C (Indirect tensile essay on cylindrical specimen IT-CY).

\section{Testing machine and sensors}

For the realization of the tests, a hydraulic press of capacity $50 \mathrm{kN}$ is used. The main Characteristics are the following ones:

- Defining the various test parameters is made using specific software.

- Creating the desired solicitation signals. The control of the press and the thermal enclosure in addition to all the necessary elements for the programming of an experiment.

The control of the press is made using the software. This program allows you to define the different parameters and create the desired solicitation signals: The control of the press and the thermal enclosure wall as well as all the necessary elements for the programming of an experiment.

\section{Instrumentation}

The measurements will take place in a room, inside a test laboratory, identified under "Room" and maintained in the ambient weather conditions.

The Modulus test machine housed in a thermoregulated enclosure calibrated/verified in extern, in which a constant test temperature between 0 and $40^{\circ} \mathrm{C}$ can be maintained near the specimens with an accuracy of $\pm 0.5^{\circ} \mathrm{C}$.

The machine is tracked metrologically by control charts obtained from the tests on a working etalon (Etalon Ring). - The deformation measuring system consisting of two linear sensors (LVDT: Linear Variable Differential Transformers), capable of measuring the transient diametral deformation of the specimen during the application of a charge pulse with an accuracy better than $1 \mu \mathrm{m}$.

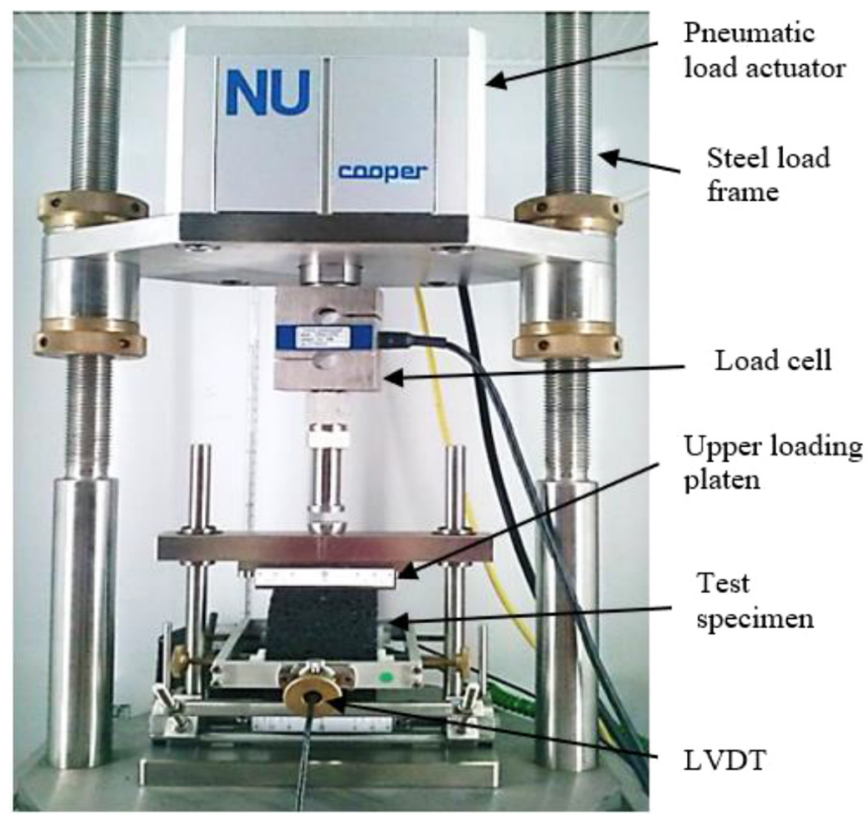

Fig. 1. Test equipment of the rigidity modulus (EN 12697-26).

- A load cell (or force sensor) for measuring the applied load with an accuracy of $2 \%$. The two force sensors and displacement are calibrated separately by an external organization.

- Adapted thermocouples, calibrated externally, able to measurement at $\pm 0.1^{\circ} \mathrm{C}$ the temperature of the specimen, and the conditions of storage and testing.

- The calibrated caliper externally, allows measuring the thicknesses and diameters of the specimens with an accuracy of $\pm 0.1 \mathrm{~mm}$.

The law of the propagation of the uncertainties of the following sensors (Force sensor, Displacement sensor LVDT, the thermocouple, caliper.) is assumed as standard Gaussian.

For the standard uncertainty of the resolution, Calibration, Drift and Sensitivity of the sensors, the Load rise time, and the Temperature, their evaluation of standard uncertainty is based on scientific judgment using all of the relevant information available.

\section{Rigidity modulus measurement}

Indirect tensile tests were conducted on compacted specimens, with a diameter of $100 \mathrm{~mm}$, a thickness of $53 \mathrm{~mm}$, and a density of $2451 \mathrm{~kg} / \mathrm{m}^{3}$ according to standard NF EN 12697-26: 2004 [4-7].

The rigidity modulus of elasticity was determined using a pneumatic press (Fig. 1) located in a thermostatic chamber to control the temperature.

The test consists of applying a cyclic compression load diametrically on the specimen. We measurement the variation of the horizontal diameter Figure 2. 


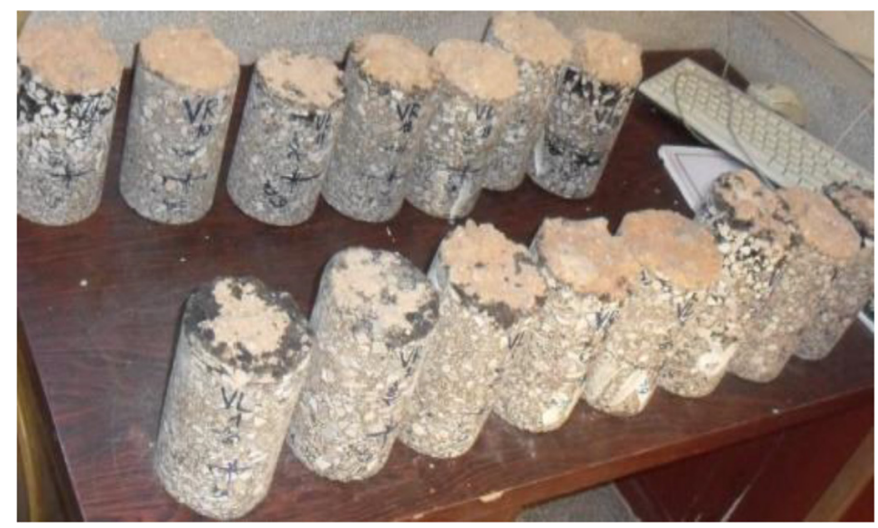

Fig. 2. Some test specimens were taken from the site.

Table 1. summarizes the conditions of the essays.

\begin{tabular}{ll}
\hline Horizontal deformation referred to & $5 \pm 2 \mu \mathrm{m}$ \\
\hline Frequency in $\mathrm{Hz}$ & 10 \\
Number of pulses & 10 \\
The pulse repetition period & $3 \pm 0.1 \mathrm{~s}$ \\
Load rise time & $124 \pm 4 \mathrm{~ms}$ \\
Poisson's ratio & 0.35 \\
Essay temperature & $15 \mathrm{C}^{\circ}$ \\
\hline
\end{tabular}

Table 2. The results of the essays.

\begin{tabular}{ll}
\hline Modulus of rigidity $(E$ in $\mathrm{MPa})$ & 6503.125 \\
\hline $\begin{array}{l}\text { Adjusted modulus of rigidity } \\
\left(E^{\prime} \text { in } \mathrm{MPa}\right)\end{array}$ & 6696.000 \\
\hline
\end{tabular}

The pulse duration of the applied load shall be 124 milliseconds, followed by a rest period of 3 seconds. The magnitude of the charge is necessary to produce a transient increase of 4 microns in the horizontal diameter of the specimen Table 1.

During the test, 10 pulses of conditioning charge are first applied to allow the press to calibrate the magnitude of the charge and the duration. Then 5 more charge pulses are applied for each direction, the variation of the diameter deformation is measured horizontal and applied load. The modulus of rigidity is calculated using the measurement of the 5 charging pulses according to the following formula [1] Table 2:

$$
E=\frac{F *(v+0.27)}{(Z * h)}
$$

where

- $E$ : is the modulus of stiffness measured, expressed in $\mathrm{MPa}$;

- F: is the value of the vertical load applied, expressed in $N$;

$-Z$ : is the amplitude of the horizontal deformation obtained during the charge cycle, expressed in $\mathrm{mm}$;

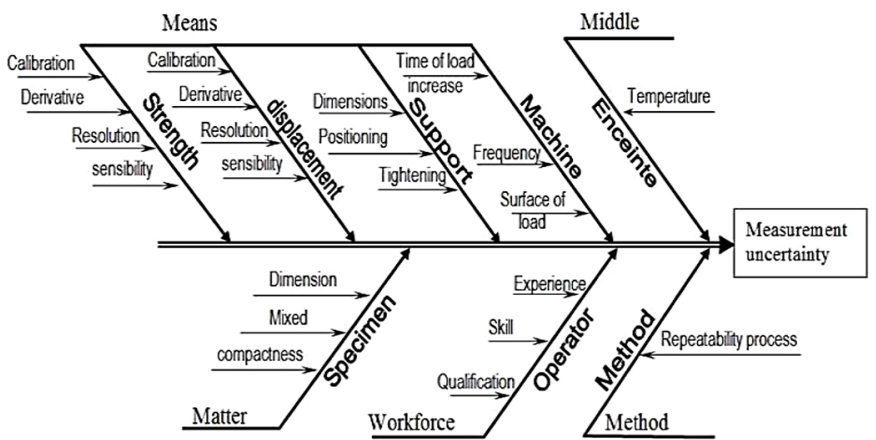

Fig. 3. The method of the $5 \mathrm{M}$.

- $h$ : is the thickness of the test piece, expressed in mm; $-v$ : is the Poisson's ratio a value of 0.35 was taken;

With,

Adjusted Modulus of rigidity given by the following formula

$$
\begin{aligned}
E^{\prime} & =E \times[1-0.322 \times(\log (E)-1.82) \\
& \times(0.6-K)] .
\end{aligned}
$$

\section{Uncertainties evaluation using GUM approach}

The structure of the uncertainty calculations follows the GUM approach $[8,9]$ and is achieved in four steps such that.

\subsection{Specify the measurand}

The definition of the measurand with the writing of the mathematical model representing it of each input quantity of the model is estimated and the value of the result (measurand) is determined.

Before starting the measurement, it is important to define what we are going to measure, we have based on the standard NF EN 12697-26: 2004 and the rigidity modulus essay technical file:

- Force $F$

- Longitudinal $Z$ displacement

- The thickness of the test specimen $h$

- The Poisson's ratio

- The load factor $k$

\subsection{Identify sources of uncertainty}

Sources of uncertainty were identified by the $5 \mathrm{M}$ method and are presented in the following diagram (Fig. 3).

The factors that can influence the value of the modulus of rigidity of the asphalt are mainly: temperature, loading speed, and stress level, frequency or loading time, sample size tested, and repeatability of the test process. All of these factors are of paramount importance to the value of the Modulus. 


\subsection{Quantify the sources}

\subsubsection{Uncertainty type A: Repeatability of the essay process $\left(u_{-} A(E)\right)$}

The complete process of determination of the Modulus $(E)$ of the material is independently made $\mathrm{n}$ time, the result is automatically obtained by the software of the piloting of the machine.

The measurement process is characterized by the estimate (experimental standard deviation) of the repeatability of

$$
E: \quad u_{-} A(E)=S_{X}
$$

where

with

$$
S_{X}=\sqrt{\frac{\sum_{n}^{i}\left(E_{i}-\bar{E}\right)^{2}}{n-1}}
$$

$$
\bar{E}=\frac{\sum E_{i}}{n}
$$

\subsubsection{Uncertainty type of the type $B:\left(u_{-} B(E)\right)$}

$$
\begin{aligned}
u_{-} B(E)= & \sqrt{\left(\frac{0.62 * u_{F}}{Z * h}\right)^{2}+\left(\frac{0.62 * F * u_{Z}}{Z^{2} * h}\right)^{2}} \\
& +\left(\frac{0.62 * F * u_{h}}{Z * h^{2}}\right)^{2}
\end{aligned}
$$

where:

- $F$ : is the value of the vertical load applied;

$-Z$ : is the amplitude of the horizontal deformation obtained during the charge cycle;

$-h$ : is the thickness of the test piece;

- $\left(u_{F}\right)$ standard uncertainty due to the charge;

- $\left(u_{Z}\right)$ standard uncertainty due to deformation;

- $\left(u_{h}\right)$ standard uncertainty due to the thickness $\mathrm{h}$ of the specimen.

with,

$$
f=E=\frac{F *(v+0.27)}{(Z * h)}=\frac{0.62 * F}{(Z * h)}
$$

and $v$ is the poisson's ratio a value of 0.35 .

Uncertainty obtained from the law of propagation of Uncertainties on the measurand equation of the modulus of rigidity we obtain the following equation $(\mathrm{MPa})$ : with:

$$
u B(E)_{(y)}=\sum_{i=1}^{n}\left[\frac{\partial f}{\partial x_{i}}\right]^{2} \cdot u^{2}\left(x_{i}\right)
$$

The correlation between the variables is negligible.

To calculate the measurement uncertainty of $u_{F}, u_{Z}$ and $u_{h}$ for each sensor, the following equation (6) is used:

$$
u=\sqrt{u_{E T^{2}}}+u_{d e r}^{2}+u_{r e s}^{2}+u_{s}^{2}
$$

where

$-u_{E T}$ : Uncertainty related to peson calibration (force sensor);

- $u_{d e r}$ : The uncertainty due to drift, (For 1st calibration we shall take $\left(u_{d e r}=u_{E T}\right)$;

$-u_{\text {res }}$ : Uncertainty related to the resolution of the measured value,

$-u_{s}$ : The uncertainty related to the sensitivity of temperature, the essays were realized only between 0 and $40^{\circ} \mathrm{C}$, at a constant temperature. At a fixed temperature, there is no drift of sensor measurement with temperature.

To determine these uncertainties, we refer to all the information (observed data, verification findings, calibration certification, expert opinion, datasheets, manufacturer's data, etc.) and in particular the following guides [JCGM -100-2008 et GUM 1995].

Uncertainty budget for the modulus of stiffness by GUM (see Tab. 3).

\subsection{Determination of expanded uncertainty}

The expanded uncertainty corresponds to a quantity defining an interval, around the result of measurement; it can be expected to include a large fraction of the distribution of values that could be reasonably attributed to the measurand

$$
U\left(E^{\prime}\right)=k \cdot u\left(E^{\prime}\right),
$$

where $K$ is the coverage factor, we can take as an coverage factor $K=2$ to have a level of confidence from $95 \%$ The final result expressed with two significant figures, by using the rule of rounding of Gauss. If all sources of uncertainty are taken into consideration, then there will be greater uncertainty [10].

Expanded uncertainty on the adjusted Modulus:

$$
U\left(E^{\prime}\right)=588.5970 \mathrm{MPa} \text {. }
$$

\section{Analysis of the contribution of the parameters}

The analysis of contribution consists of identifying among all the parameters of the model those who influence the essays of the Modulus of rigidity.

To determine the most influential parameters, the contribution analysis is used to identify the input parameters that contribute the most to the uncertainty of the final results. They are characterized by uncertainty and high sensitivity. However, a parameter with excessive sensitivity and small uncertainty, or vice versa, can be a significant contributor.

The contributions of the parameters are represented in the form of a histogram.

According to the analysis of this histogram, we find that repeatability has a contribution of around $45.23 \%$ represents the highest percentage index, compared to other factors (force sensor, LVDT displacement sensors, and the thickness of the specimen), this is explained by the values of the uncertainties of these factors which are negligible Figure 4. 


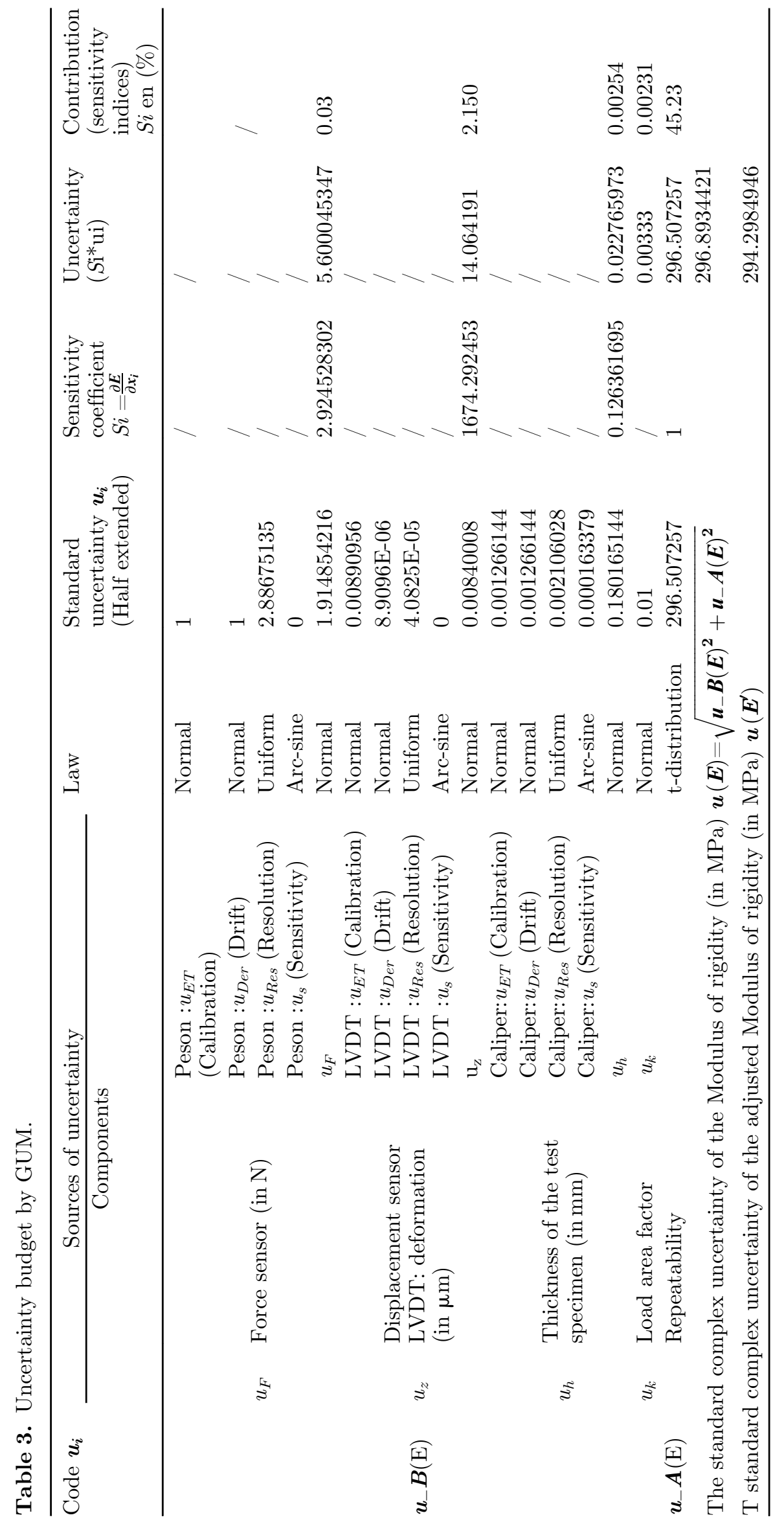


Table 4. Density of distributions for the input variables.

\begin{tabular}{lll}
\hline Source of uncertainty & Type of distribution & standard uncertainty \\
\hline Force sensor $\boldsymbol{u}_{\boldsymbol{F}}$ en $(\mathrm{N})$ & Normal & 1.914854216 \\
Deformation $\boldsymbol{u}_{\boldsymbol{z}}$ en $(\boldsymbol{\mu m})$ & Normal & 0.00840008 \\
Thickness of the test specimen $\boldsymbol{u}_{\boldsymbol{h}}$ in $(\mathrm{mm})$ & Normal & 0.180165144 \\
Load area factor $\boldsymbol{u}_{\boldsymbol{k}}$ & Normal & 0.01 \\
Repeatability $\boldsymbol{u}_{-} \boldsymbol{A}(\mathrm{E})$ & t-distribution & 296.507257 \\
\hline
\end{tabular}

\section{Contribution (\%)}

\begin{tabular}{c|c}
$\begin{array}{c}\text { Load area factor } \\
\text { uk }\end{array}$ & $0.00231 \%$ \\
$\begin{array}{c}\text { Thickness of the } \\
\text { test specimen uh } \\
\text { Force sensor uF }\end{array}$ & $0.00254 \%$ \\
$\begin{array}{c}\text { Deformation uz } \\
\text { Repeatability } \\
\text { u_A(E) }\end{array}$ & $2.15 \%$ \\
\hline
\end{tabular}

$45.23 \%$

Fig. 4. The contributions of the parameters.

\section{Uncertainties evaluation using Monte Carlo}

Monte Carlo Simulation (MCS) is the second method to estimate the uncertainty of measurements, where evaluating the measurement uncertainty by the MCS method can be carried out by establishments of the model equation for the measurand in the function of the individual parameters of influence, then selecting the significant sources of uncertainty, identification of the probability density functions corresponding to each source of uncertainty selected, and selecting the number Monte Carlo trials, and finally calculating the $\mathrm{M}$ results by applying the equation that was defined for the measurand [11] Figure 5.

The Monte Carlo numerical method [12-14] then is a necessary alternative. To implement it, we must first define the factors influencing the output quantity and associate them via an analytical or numerical model. It may be noted that this step was already necessary for the linear method. Each factor of influence is associated with a statistical distribution. A random generator is then used to generate prints of each influence factor.

The probability densities of each input quantity are defined in Table 4 of the GUM method. The standard deviations of each variable are obtained by taking the standard uncertainties calculated by the GUM approach.

When all the distributions of the input quantities have been defined, we can then generate realizations of each input quantity by drawing in their probability density

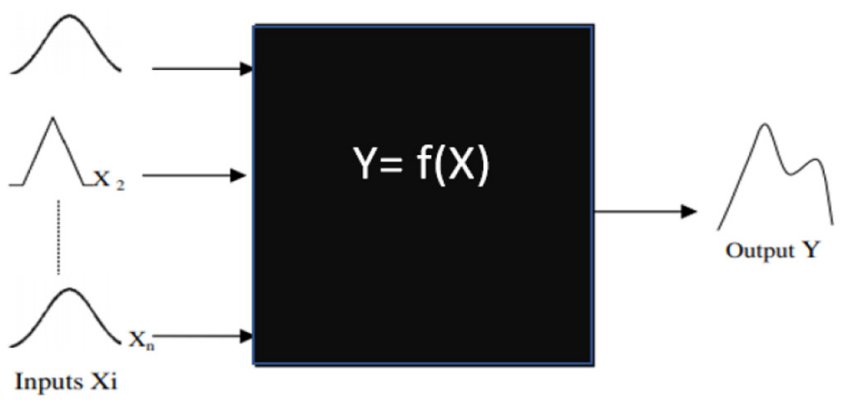

Fig. 5. Propagation of uncertainty model.

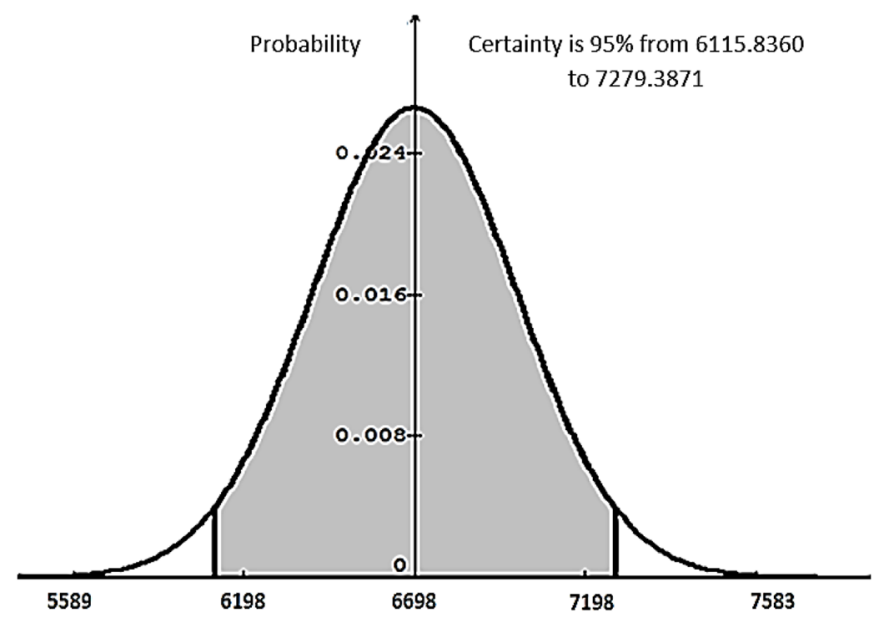

Fig. 6. Result of the Monte Carlo simulation.

function, it is necessary to have a sufficiently powerful random number generator, the calculation is carried out in a specific software (MC-Ed) It allows to define a Modulus for each Modulus size (load, strain, specimen thickness, load surface factor, etc.) and then assembles them into a Meta-Modulus. Finally, he chooses the numerical value of each term of the model, its associated uncertainty, and the probability function.

The Monte Carlo simulations of values obtained from the output quantity, which makes it possible to construct the empirical distribution of the measurand Figure. 6.

We notice that the curve represents the Gaussian distribution, the maximum stiffness modulus value (7279.3871 $\mathrm{MPa})$, and the minimum (6115.8360 MPa) define an interval that contains $95 \%$ of the values 
Table 5. Comparison results.

\begin{tabular}{lll}
\hline Grandeur & Method GUM & Method Monte-Carlo \\
\hline$E^{\prime}$ & $6696 \mathrm{MPa}$ & $6698.34 \mathrm{MPa}$ \\
$\boldsymbol{u}$ & $294.2984 \mathrm{MPa}$ & $288.5634 \mathrm{MPa}$ \\
Extended interval in MPa & {$[6107.4030 ; 7284.5970](K=2)$} & {$[6115.8360 ; 7279.3871]$ (of 95\%) } \\
\hline
\end{tabular}

attributable to the measurand. The Mean value is $6698.34 \mathrm{MPa}$ and the standard deviation is $288.5634 \mathrm{MPa}$.

Finally, the result is given with a confidence interval $I p$ $\left(E^{\prime}\right)=\left[E^{\prime}{ }_{\text {low }}, E^{\prime}{ }_{\text {high }}\right]$ corresponding to a probability $p$ of 95\% (Fig. 6):

- Standard deviation $=288.5634 \mathrm{MPa}$

- Extended interval, $\mathrm{I}_{95 \%}\left(E^{\prime}\right)=[6115.8360 ; 7279.3871]$ MPa.

\section{Comparison of the results given by the two methods}

The results obtained by the GUM method and by the Monte-Carlo method for the evaluation of measurement uncertainties of the modulus of rigidity.

We note that the difference between the two confidence intervals obtained is very small and that the interval calculated by numerical simulation of Monte-Carlo is included in that of the propagation of the uncertainties. Moreover, these results are quite in agreement with each other and we can consider that the results given by the numerical simulation of Monte Carlo validate the law of propagation of the uncertainties.

We also applied the comparison criterion described in the document (BIPM JCGM 10). Indeed, it is advisable to apply the GUM method by adding terms of higher degree if necessary and to give a confidence interval of $95 \%$ for the output variable. Then, to validate the GUM method, it is necessary to apply the numerical simulation of Monte Carlo in order to provide a standard uncertainty and a confidence interval of $95 \%$. It must then be determined whether the confidence intervals obtained by the Monte Carlo method and the law of propagation of the uncertainties are in agreement with a degree of approximation calculated as follows Table 5 .

We express the standard deviation in the form $a \times 10^{r}$ or $\mathrm{a}$ is an integer of 2 numbers (corresponding to the number is the significance level, here 2) and $r$ an integer. The precision othe comparison is then: $\delta=0.5 \times 10^{r}$.

The expanded uncertainties obtained by each method are compared. We form the differences:

$$
\begin{aligned}
& d_{\text {low }}=\left|E^{\prime}-U_{(G U M)}\left(E^{\prime}\right)-E_{\text {low(MONTE-CARLO })}^{\prime}\right| \\
& d_{\text {high }}=\left|E^{\prime}+U_{(G U M)}\left(E^{\prime}\right)-E_{\text {high(MONTE-CARLO) }}^{\prime}\right|
\end{aligned}
$$

If these differences are both less than the previously determined tolerance, then the results obtained by the GUM method are validated.
If we take 2 significant figures for, $u_{\text {MONTE-CARLO }}$ we have: $E^{\prime}=6696 \mathrm{MPa}$

$$
u_{\text {MONTE-CARLO }}=288 \mathrm{MPa}=28.10^{1} \mathrm{MPa}
$$

and

$$
\begin{gathered}
\delta=0.5 \times 10^{1}=5 \\
d_{\text {low }}=1.78621 \\
d_{\text {high }}=2.78324
\end{gathered}
$$

The results obtained show that the two quantities $d_{\text {low }}$ and $d_{\text {high }}$ are not bigger than $\delta$, so the analytic approach GUM is validated by the approach Monte-Carlo.

\subsection{Comparison of two variances $u^{2}$ (Monte _ Carlo) and $u^{2}$ (GUM) (Fisher's test)}

The Fisher comparison test compares the variances of two uncertainty calculation approaches (GUM) and the numerical simulation of Monte Carlo under populations, and confronts the following hypotheses:

$$
\begin{array}{ll}
H_{0}: & u^{2}{ }_{(\text {MONTE_CARLO })} \approx u^{2}{ }_{(G U M)} \\
H_{1}: & u_{(\text {MONTE_CARLO })}^{2} \neq u_{(G U M)}^{2}
\end{array}
$$

The statistic in $F$ test is thus defined by

A risk of $\alpha=0.05(5 \%)$

In our case $F=\frac{u^{2}{ }_{\text {GUM })}}{\left.u^{2} \text { (MONTE_CARLO }\right)}$;

The degrees of freedom:

$$
\begin{gathered}
n_{\mathrm{GUM}}-1=6 \\
n_{\text {MONTE CARLO }}-1=6
\end{gathered}
$$

We calculate:

$$
F=\frac{u_{(\mathrm{GUM})}^{2}}{u_{\left(\mathrm{MONTE} \_\mathrm{CARLO}\right)}^{2}}=\frac{(294.2984)^{2}}{(288.5634)^{2}}=1.0401 .
$$

We compare this value to the tabular value of the $F$ in the table of the law $F$ Table 6 :

$$
F_{n_{\mathrm{MONTE} \mathrm{CARLO}}-1}^{n_{\mathrm{GUM}^{-1}}}=F_{6}^{6}=4.284
$$


Table 6. Extract from the Fisher table.

\begin{tabular}{lllllllll}
\hline & \multicolumn{7}{c}{$\mathrm{F}_{.05}\left(v_{1}, v_{2}\right)$} \\
\cline { 2 - 8 }$v_{2} \backslash v_{1}$ & 1 & 2 & 3 & 4 & 5 & 6 & 7 & 8 \\
\hline 1 & 161.448 & 199.500 & 215.707 & 224.583 & 230.162 & 233.986 & 236.768 & 238.882 \\
2 & 18.513 & 19.000 & 19.164 & 19.247 & 19.296 & 19.330 & 19.353 & 19.371 \\
3 & 10.128 & 99.552 & 9.277 & 9.117 & 9.013 & 8.941 & 8.887 & 8.845 \\
4 & 7.709 & 6.944 & 6.591 & 6.388 & 6.256 & 6.163 & 6.094 & 6.041 \\
5 & 6.608 & 5.786 & 5.409 & 5.192 & 5.050 & 4.950 & 4.876 & 4.818 \\
6 & 5.987 & 5.143 & 4.757 & 4.534 & 4.387 & 4.284 & 4.207 & 4.147 \\
7 & 5.591 & 4.737 & 4.347 & 4.120 & 3.972 & 3.866 & 3.787 & 3.726 \\
\hline
\end{tabular}

It is concluded that the observed ratio is smaller than the reference ratio: $F<F_{6}^{6}$. and so we do not reject the hypothesis $H_{0}$.

\section{Conclusion and recommendation}

The analytical method (GUM) makes it possible to easily perform a complete analysis of uncertainty: it makes it possible to calculate the uncertainty of the impact scores, their sensitivity to the variation of the input parameters, and the contribution of the uncertainty of the test. The modulus of stiffness according to GUM the greatest uncertainty comes from the Repeatability.

The comparison between the uncertainty calculated using the analytical method and the results of the Monte Carlo method showed no significant difference, it is because of the linearity of the measurement models used for each of the tests and the independence of the effects of the contributions to uncertainty considered.

The Monte Carlo simulations carried out made it possible to show that the modulus of rigidity is Gaussian so it is easy to give a confidence interval, with the maximum value of modulus of rigidity (7279.3871 $\mathrm{MPa})$ and the minimum (6115.8360 MPa) define an interval that contains $95 \%$ of the values attributable to the measurand. The Mean value is $6698.34 \mathrm{MPa}$ and the standard deviation is 288.5634 MPa.

Certain functionality of Monte Carlo is the reduction of the effort of analysis required for the complex or not linear models, in particular since the partial by-products of the first one(night) or the upper order used to supply coefficients of sensibility for the law of distribution of the uncertainty (GUM) are not necessary.

The analytical method remains very relevant as far as the cases of nonlinearity are rare in the practical order of magnitude of the sources of uncertainties and in the complex processes.

Both methods are thus feasible and it is interesting to make these two calculations in parallel to verify his result.
Mainly, for the evaluation of the uncertainties of test measurement of stiffness modulus. It is recommended to proceed with the GUM approach. The ease of use, speed, and the amount of information essential to the analysis of the uncertainty it provides. Making this method available would allow the uncertainty analysis tgn popularity, improving the interpretation of rigidity modulus test results.

The use of the analytical method (GUM) would, in addition, improve the quality of the uncertainty analyzes, since the sensitivity quickly reveals the incoming parameters likely to contribute a lot to the uncertainty of the impact scores.

\section{References}

1. EN 12697-26:2012, Bituminous mixtures, Test methods for hot mix asphalt -Annex c: Stiffness (2012)

2. C. Huet, Modulus representation and complex arithmetic and logarithmic complacencies, Tome 1-5 (1947)

3. P. Cornil, Determination of the modulus of rigidity and bending fatigue tests on test specimens of hydrocarbon materials embedded at one end Rapp rech, CRR Bruxelles (1968)

4. GM, International vocabulary of metrology, Basic and general concepts and associated terms (VIM), BIPM, JCGM 200:2008 (2008)

5. H. Di Benedetto, M.N. Partl et al., Stiffness testing for bituminous mixtures, Mater. Struct. 34, 66-70 (2001)

6. Y.R.Y. Seo Kim et al., Dynamic modulus testing of asphalt concrete in indirect tension mode, Transportation Research Record 1891, 163-173 (2004)

7. M.E. Nunn, T.M. Smith, Improvements to the indirect tensile stiffness modulus test. 2nd European Symposium Performance and durability of bituminous materials, Leeds (1997)

8. ISO/IEC GUIDE 98-3:2008 Uncertainty of measurement Part 3: Guide to the expression of uncertainty in measurement (GUM: 1995)

9. F. PLATEL, Putting Methods into Practice. Monte-Carlo Projet Monte-Carlo Editor (MC-Ed), MetGen, Dossier métrologie 25 
10. ISO/CEI Guide 25, General requirements for the competence of calibration and testing laboratories (1990)

11. JCGM, Evaluation of measurement data-supplement 1 to the Guide to the expression of the measurement propagation of distributions using a Monte-Carlo method, BIPM. JCGM $101(2008)$

12. E. GENTLE James, Random Number Generation and Monte Carlo methods - Springer, Statistics and Computing, second Edition (2003)
13. H. Schwenke, B.R.L. Siebert, F. Wäldele, H. Kunzmann, Assessment of uncertainties in dimensional metrology by monte carlo simulation: proposal of a modular and visual software, Ann. CIRP (2000)

14. ISO/IEC Guide 98-3/Suppl.1:2008(E) Uncertainty of measurement - Part 3: Guide to the expression of uncertainty in measurement (GUM: 1995) Supplement 1: Propagation of distributions using a Monte Carlo method. Geneva (Switzerland): ISO, December (2008)

Cite this article as: Hicham Mezouara, Latifa Dlimi, Abdelouahhab Salih, Mohamed Afechcar, Houcine Zniker, Evaluation of the measurement uncertainty of the stiffness modulus: test case of indirect tensile on cylindrical specimens, Int. J. Metrol. Qual. Eng. $\mathbf{1 2}, 8(2021)$ 